\title{
Reflections on the effects of nitric oxide produced by a new dressing in the local management of diabetic foot ulcers
}

\author{
Yolanda García-Álvarez, José Luis Lázaro-Martínez, Raúl Juan Molines-Barroso \\ Diabetic Foot Unit, Universidad Complutense de Madrid, Instituto de Investigación Sanitaria del Hospital Clínico San Carlos (IdISSC), Madrid, \\ Spain \\ Correspondence to: Prof. Dr. José Luis Lázaro-Martínez. Diabetic Foot Unit, Universidad Complutense de Madrid, Edificio Facultad de Medicina, \\ Pabellón 1, Avda, Complutense s/n, Madrid 28040, Spain. Email: diabetes@ucm.es. \\ Provenance: This is an invited Editorial commissioned by the Section Editor Kaiping Zhang (AME College, AME Group, China). \\ Comment on: Edmonds ME, Bodansky HJ, Boulton AJM, et al. Multicenter, randomized controlled, observer-blinded study of a nitric oxide \\ generating treatment in foot ulcers of patients with diabetes-ProNOx1 study. Wound Repair Regen 2018;26:228-37.
}

Submitted Nov 13, 2018. Accepted for publication Nov 13, 2018.

doi: $10.21037 /$ atm.2018.11.34

View this article at: http://dx.doi.org/10.21037/atm.2018.11.34

A diabetic foot ulcer (DFU) precedes $75-85 \%$ of amputations in people with diabetes (1). The estimated global prevalence of DFU is $6.3 \%$ (2), with a lifetime incidence between $19 \%$ and $34 \%$ in people with diabetes. Nonhealing ulcers increase the probability of infection and amputation, which severely decrease life expectancy and increase costs. The treatment of diabetic foot complications reportedly may exceed the treatment costs of many common cancers (3).

High levels of nitric oxide (NO) metabolites have shown a positive regulatory effect on the repair process of wound healing, while inhibition of NO production is related to impaired healing (4). However, the mechanism of action of $\mathrm{NO}$ on wound healing remains unclear. Diabetes contributes to the decreased formation of $\mathrm{NO}$ metabolites in the wound environment. EDX110 is a new dressing system comprising two layers that allow the generation of NO in situ and also enable adequate moisture management and facilitate debridement.

In a recent publication (5), the ProNOx1 study, a multicentre (10 hospitals from the UK), open-label, randomized controlled trial showed the safety and efficacy of EDX110 (Edixomed, London, UK) in comparison with the standard of care (SOC) in the treatment of a "real world" foot ulcer population. One hundred and thirty-five participants with 148 chronic ulcers ( $>6$ weeks of evolution) were treated for 12 weeks. The co-primary outcomes were the percentage ulcer area reduction (PAR) at 12 weeks and the tolerability and safety of EDX110. The participants were randomly assigned (1:1) to either the EDX110 group, which they themselves placed every 48 hours, or the control group, in which the investigators provided treatment with whichever available dressing they considered best clinical practice for their participants at that time.

The findings showed that EDX110 achieved a median PAR of $88.6 \%$ compared to $46.9 \%$ in the control group after 12 weeks $(\mathrm{P}=0.016)$. Wound size reduction in the EDX110 group at 4 weeks was similar to that achieved by the control group at 12 weeks. Participants with an ulcer sized $\geq 1 \mathrm{~cm}^{2}$ and those with an ulcer duration $\leq 6$ months showed the greatest beneficial effect after EDX110 application relative to that of the control group. Additionally, there were no serious adverse events related to EDX110 or SOC and EDX110 treatment was well tolerated.

The evidence base for the stimulation of DFU healing is weak, and good-quality research relating to routine clinical care is needed (6). After this judgment, in conjunction of the European Wound Management Association and the International Working Group of the Diabetic Foot, several initiatives have been promoted and recently two high-quality RCTs have been published with important achievements in the field of DFU management $(7,8)$. The results of current research along with the findings of previous properly-designed trials aim to improve the management of foot ulcers. In this case, an intention-totreat analysis, observer-blinded or randomization strategy unaware from centers was reported as relevant points of a high standard. However, some differences can be observed between trial designs. 
All three investigations provided appropriate debridement of nonviable tissue and adequate off-loading pressure relief of the ulcers during the follow-up, which represent the central tenet of any treatment plan for neuropathic DFU (9). However, the evidence of effectiveness differs between the methods of debridement currently available to community healthcare professionals and in most cases, the selection of debridement method is based on the available expertise (10). Standardization of debridement methods and frequency seems not to have been controlled in this study including 10 different hospital sites in which the investigators could alter the dressing type as clinically required.

Something similar occurred with off-loading devices. The efficacy of different pressure-relieving interventions has demonstrated varying effects on healing rates (11). Edmonds and colleagues aimed to study a "real world population" as one of the main strengths of the study and included neuropathic, infected, and ischemic DFU. However, this also implies a dispersion of the sample. One of the leading factors to consider when prescribing the off-loading device is the causal aetiology of the ulcer (9). Standardization of off-loading processes emphasizes the real effect of the $\mathrm{NO}$-generating dressing in comparison with the control group. Furthermore, specialists working in DFU care centers require knowledge of which dressing is optimized for healing ulcers according to their aetiology. Although a sub-analysis was performed related to ulcer size and time of evolution, the "real effect" per the causal aetiology should enhance the indication of the NOgenerating dressing.

Clinicians should interpret the findings of the NOgenerating treatment in the context of the study and its external validation. The control group was treated in 10 hospitals in the same country, which means that this control group was treated under the policies of a unique healthcare system. A multicenter trial in several countries, similar to other trials $(7,8)$, should improve the external validation of the results.

The absolute effect of $\mathrm{NO}$ is difficult to determine after reading this publication because the levels of $\mathrm{NO}$ in the wounds were not measured. NO generation is lost or impaired in patients with diabetes (12). Despite this, participants with poor diabetes control did not have a different response to the treatments than those with normalized glycemic control. Furthermore, the control group was not treated with the same control dressing. Despite the fact of the absolute effect of NO could not be measured, the study has demonstrated that the application of EDX110 enhances the percentage area reduction better than that of the SOC.

Participants in the study were directed to change the EDX110 themselves and the findings of tolerability and adverse events in comparison with those in the control group demonstrated the easy and secure applicability of the two-layer dressing system. The application system, together with these encouraging results, suggests that EDX110 could become in a useful therapy to be adopted by clinicians, although the results of a costs analysis are awaited.

Therefore, the ProNOx1 study is a good-quality research with promising results regarding DFU healing. Although the origin of the effect cannot be ensured, EDX110 enhances the best available SOC, in a nonspecific population affected by DFU without increasing adverse events or differing from the easy applicability of the common dressing system. Nevertheless, the specific indications per the causal aetiology of the DFU and treatment costs analysis need to be defined.

The authors considered this dressing a "universal dressing" that could facilitate the first treatment of DFUs of different etiologies. Maybe it could be useful because it could allow for faster therapeutic decision-making at the beginning of treatment, with more specialized treatment performed later. Hopefully, this will help to improve the healing and reduce complications of ulcers in the diabetic foot.

\section{Acknowledgements}

None.

\section{Footnote}

Conflicts of Interest: The authors have no conflicts of interest to declare.

\section{References}

1. Boulton AJ, Vileikyte L, Ragnarson-Tennvall G, et al. The global burden of diabetic foot disease. Lancet 2005;366:1719-24.

2. Zhang P, Lu J, Jing Y, et al. Global epidemiology of diabetic foot ulceration: a systematic review and metaanalysis (dagger). Ann Med 2017;49:106-16.

3. Armstrong DG, Boulton AJM, Bus SA. Diabetic Foot Ulcers and Their Recurrence. N Engl J Med 2017;376:2367-75.

4. Witte MB, Barbul A. Role of nitric oxide in wound repair. 
Am J Surg 2002;183:406-12.

5. Edmonds ME, Bodansky HJ, Boulton AJM, et al. Multicenter, randomized controlled, observer-blinded study of a nitric oxide generating treatment in foot ulcers of patients with diabetes-ProNOx1 study. Wound Repair Regen 2018;26:228-37.

6. Jeffcoate WJ, Bus SA, Game FL, et al. Reporting standards of studies and papers on the prevention and management of foot ulcers in diabetes: required details and markers of good quality. Lancet Diabetes Endocrinol 2016;4:781-8.

7. Edmonds M, Lazaro-Martinez JL, Alfayate-Garcia, et al. Sucrose octasulfate dressing versus control dressing in patients with neuroischaemic diabetic foot ulcers (Explorer): an international, multicentre, double-blind, randomised, controlled trial. Lancet Diabetes Endocrinol 2018;6:186-96.

8. Game F, Jeffcoate $W$, Tarnow L, et al. LeucoPatch system for the management of hard-to-heal diabetic foot ulcers

Cite this article as: García-Álvarez Y, Lázaro-Martínez JL, Molines-Barroso RJ. Reflections on the effects of nitric oxide produced by a new dressing in the local management of diabetic foot ulcers. Ann Transl Med 2018;6(Suppl 2):S101. doi: 10.21037/atm.2018.11.34 in the UK, Denmark, and Sweden: an observer-masked, randomised controlled trial. Lancet Diabetes Endocrinol 2018;6:870-8.

9. Armstrong DG, Lavery LA, Nixon BP, et al. It's not what you put on, but what you take off: techniques for debriding and off-loading the diabetic foot wound. Clin Infect Dis 2004;39 Suppl 2:S92-9.

10. Elraiyah T, Domecq JP, Prutsky G, et al. A systematic review and meta-analysis of debridement methods for chronic diabetic foot ulcers. J Vasc Surg 2016;63:37S-45S.e1-2.

11. Lewis J, Lipp A. Pressure-relieving interventions for treating diabetic foot ulcers. Cochrane Database Syst Rev 2013;(1):CD002302.

12. Williams SB, Cusco JA, Roddy MA, et al. Impaired nitric oxide-mediated vasodilation in patients with noninsulin-dependent diabetes mellitus. J Am Coll Cardiol 1996;27:567-74. 